\title{
Twitter and Society
}

Katrin Weller, Axel Bruns, Jean Burgess, Merja Mahrt, and Cornelius Puschmann

Title: Twitter and Society

Publisher: Peter Lang, 2014

ISBN: 978-1-4331-2169-2

447 pages

At a time when many media scholars were turning their attention to evolving technologies and their role in communication, Philip Napoli (2011) urged researchers across disciplines to consider not only the newness of innovations but also their impacts on the ways people communicate and engage with one another. More universal examinations of technologies and their users, he argued, were necessary to understand the sociocultural consequences of technological adoption.

Napoli's call has since been taken up by a collage of scholars and practitioners, each offering unique and salient contributions to media and communication studies and beyond. Crisis communication, health communication and informatics, linguistics, pedagogy, information science, and a diversity of other areas have increasingly been examined against the backdrop of change brought about by digital advancements.

These studies are important not only for their navigation of uncharted waters, but also for their ability to highlight the relevance of emerging media technologies across a swath of scholarship and practice. Indeed, platforms such as Twitter have grown from ways to keep one other updated to more powerful connective tools where relationships and networks live and die by communicative action.

Twitter and Society recognizes that growing clout, one that Twitter in particular has harnessed over its eight years of public existence. Here is an edited volume that feels at times less like a collection of research articles and more like a window into the crunching gears that drive society's fascination with, and growing reliance on, a select number of social network sites (SNSs) such as

The Journal of Media Innovations 1.1 (2014): 134-137

(C) Avery E. Holton 2014

http://www.journals.uio.no/index.php/TJMI 
Twitter, which now claims more than 200 million unique daily users across the globe, collectively producing more than a billion pieces of content every 48 hours.

Rather than positioning itself as a book geared toward scholars-which it undoubtedly is at times-Twitter and Society forges an all-important bridge between academia and practice, offering a book in two parts. The first section explicates concepts and methods that have driven Twitter as a technological and sociological instrument, along with an overview of the current state of research surrounding it. The second section then works to strip away any academic silos by offering a collage of insightful essays centered on culture, privacy, and information preservation, as well as the uses and implications of Twitter in popular culture, brand communication, politics and activism, journalism, crisis communication, and academia.

Collectively, the authors set out to strategically unpack Twitter by first critiquing current approaches to Twitter research and offering new, more thoughtful and analytical methods. They then lean into the central thesis of the book: the idea that Twitter is not merely a technological artifact with mass media implications, but rather a mechanism through which society can and has become bound in ways never before imagined. Indeed, it "has become a backbone for a much wider range of manual and automated communicative exchanges,"that increasingly affect individuals and institutions on a global scale (Puschmann, Bruns, Mahrt, Weller \& Burgess, 2014, pp. 430).

In order to understand the root of current and future impacts of Twitter on society and how they might be analyzed, the authors begin in the first 10 chapters by questioning Twitter's role as a private and public space for individuals and institutions before challenging the current state of Twitter research to consider new and mixed methods of analysis. In his opening chapter, Jan-Hinrick Schmidt describes the creation process of "personal publics," wherein individual users select content to share and engage in based on personal relevance and with a select audience in mind. These publics, Schmidt duly argues, have helped to reconstruct media practices (e.g., inviting individuals into the news process, encouraging journalists to include personal elements in their tweets) and have opened doors for social inclusion. At the same time, information that once may have been kept private has become much more public thanks to the affordances of Twitter.

This raises questions about how individuals decide what is public versus private. Axel Bruns, Hallvard Moe, and Alexander Halavais examine those questions, noting that users work through macro-, meso-, and micro-publics by employing particular mechanisms built into Twitter. Individuals use hashtags to engage in specific topics (macro), develop follower networks (meso), and have more interpersonal and private relationships through the use of replies and direct messages (micro). Collectively, these create an intricate structure wherein individuals can observe the action of others and make decisions about what content to post and what mechanisms to post it with based on its intended 
publicness or privateness.

In order to address these and related concerns, the authors offer a mix of methodologies that branch out from the more popular social network analysis (SNA) that began emerging before the turn of the decade. Most notably, they build a case for coupling network and structural analytics with traditional and novel forms of content analysis to discover not only which individuals are connected to which and through what channels and networks, but also how and why exchanges of new information take place, what they look like, and what they can tell us about the functionality of digital social networks.

In her chapter on qualitative methods for Twitter research, Alice Marwick notes that quantitative analyses may present rich data pools from which to establish network connections and changes brought on by temporality. Yet, in order to more fully understand what drives individuals to exchange information on Twitter, to become part of digital discourses, and to opt in and out of networked communities, qualitative approaches such as interviews, ethnographic fieldwork, and textual analysis should not be shuttered away.

This cautious throwing open of methodological doors carries over into the second section of the book, which begins by giving more attention (and arguably more importance) to the affects Twitter can have on individuals and institutions, is divided into two sections, perspectives and practices; the latter is an expansive compilation of 15 chapters. The former dances through a series of important functions and concerns related to Twitter, including geotagging, message automation and archiving, and the development of offline culture through Twitter exchanges. Yet, it is a chapter on privacy penned by Michael Zimmer and Nicholas Proferes that finds bits of itself embedded in nearly every chapter that follows. Because privacy has become much more of a process than a luxury on Twitter, it is a constant, if not growing, concern among users.

These users, though, continue to meld Twitter to fit their expectations, using it for far more than a means to share nuggets of ephemeral news, as Richard Rogers points out in his foreword. As the authors explain, Twitter is now being used to create digital fandom and to infiltrate the once impenetrable walls of professional sports teams' public relations. It is being crafted as a means for political engagement and social movements. Individuals and institutions are embedding themselves within Twitter with the hopes of creating brand awareness for themselves just as they are using the space to share real-time information about health and crisis situations.

Such a diversity of uses brings with it a need to examine Twitter less as a technological artifact and more as an agent of digital and social change wherein the public, both private and unrestricted, has governed the development and uses of the platform. Such a communal distribution of power will no doubt continue to drive the functions of Twitter and its predecessors. Understanding how to approach the current and coming changes scientifically, ethically, and holistically is tantamount to anticipating these transformations themselves. 
Collectively, the authors and editors of Twitter and Society have persuasively offered Twitter as a technological mechanism of social and cultural change wherein scholars and practitioners are uniquely positioned to both reimagine and refashion how Twitter is researched and how it is best put to use as it continues to lay a foundation for digital exchange and communality.

Avery E. Holton

Assistant Professor

University of Utah, Department of Communication

\section{References}

Napoli, P. M. (2011). Audience evolution: New technologies and the transformation of media audiences. New York: Columbia University Press.

Puschmann, C., Bruns, A., Mahrt, M., Weller, K., \& Burgess, J. (2014). Epilogue: Why study Twitter? In K. Weller, A. Bruns, J. Burgess, M. Mahrt \& C.

Puschmann (Eds.), Twitter and Society (pp. 425-432). New York: Peter Lang. 